\title{
Acidentes de trabalho e os riscos ocupacionais identificados no Serviço de Atendimento Móvel de Urgência*
}

\author{
Work accidents and occupational risks identified in the Mobile Emergency Service \\ Accidentes de trabajo y riesgos ocupacionales identificados en \\ el Servicio de Atendimiento Móvil de Urgencia
}

Como citar este artigo:

Goulart LS, Rocha LP, Carvalho DP, Tomaschewski-Barlem JG, Dalmolin GL, Pinho EC. Work accidents and occupational risks identified in the Mobile Emergency Service. Rev Esc Enferm USP. 2020;54:e03603. doi: https://doi.org/10.1590/S1980-220X2018056903603

\section{Leonardo Salomão Goulart ${ }^{1}$}

Laurelize Pereira Rocha ${ }^{1}$

Deciane Pintanela de Carvalho ${ }^{1}$

Jamila Geri Tomaschewski-Barlem ${ }^{1}$

Graziele de Lima Dalmolin²

Eliana Cardia de Pinho ${ }^{3}$

* Extraído da dissertação: "Riscos ocupacionais e acidentes de trabalho entre trabalhadores do Serviço de Atendimento Móvel de Urgência (SAMU 192): um estudo no estado do Rio Grande do Sul”, Programa de Pós-Graduação em Enfermagem,

Universidade Federal do Rio Grande, 2018.

${ }^{1}$ Universidade Federal do Rio Grande, Rio Grande, RS, Brasil.

${ }^{2}$ Universidade Federal de Santa Maria, Santa Maria, RS, Brasil.

${ }^{3}$ Instituto Federal de Educação, Ciência e Tecnologia do Rio Grande do Sul, Rio Grande, RS, Brasil.

\begin{abstract}
Objective: To analyze the occurrence of work accidents among Mobile Emergency Service workers and the association with the identified occupational risks. Method: A quantitative, exploratory, descriptive and cross-sectional study conducted with Mobile Emergency Service workers in Rio Grande do Sul, between January 2016 and November 2017, using an online instrument. Results: A total of 265 workers participated. There was a significant association between the occurrence of accidents at work and professional category $(\mathrm{p}=$ 0.041); as well as the occurrence of work accidents and the mesoregion ( $p=0.015)$. In the significant associations between the occurrence of accidents at work and occupational risks, accidents with sharps, physical aggression, animal bites, verbal aggression, traffic accidents during commuting and falls were highlighted. In addition, a significant association between the occurrence of an accident at work and work leave $(p=0.000)$ was found. Conclusion: The occurrences of work accidents during pre-hospital service activities are related to the professional category of workers, the work mesoregion, work leave and the exposure of workers to different occupational risks.
\end{abstract}

\section{DESCRIPTORS}

Accidents, Occupational; Emergency Medical Services; Ambulances; Occupational Risks; Occupational Health.

Autor correspondente:

Deciane Pintanela de Carvalho

Rua General Osório, s/n

CEP 96200-190 - Rio Grande, RS, Brasil

Recebido: $14 / 01 / 2019$

deciane.carvalho@gmail.com 


\section{INTRODUÇÃO}

As ações de cuidado prestados pelos trabalhadores de saúde no ambiente pré-hospitalar apresentam-se repletas de situações favoráveis ao adoecimento e a ocorrência de acidentes de trabalho ${ }^{(1)}$. A exposição do trabalhador ao acidente de trabalho oriundo da presença de diferentes riscos ocupacionais ocorre em diferentes momentos do atendimento, seja na assistência direta ao paciente ou até mesmo ao se envolver em um acidente de trajeto durante o deslocamento da equipe até o local onde o atendimento será realizado ${ }^{(1-2)}$.

No Brasil, este tipo de assistência é realizada pelo Serviço de Atendimento Móvel de Urgência (SAMU), que busca atender as situações de agravo à saúde da população em condições de urgência e emergência nos mais diferentes locais, conferindo uma melhor resposta às situações que oferecem risco de morte e que possam reduzir as sequelas decorrentes do adoecimento ${ }^{(3)}$. O trabalho no ambiente pré-hospitalar compreende características como complexidade das funções executadas, ritmo acelerado, condições inapropriadas de trabalho, os quais podem gerar riscos ocupacionais, ocorrência de acidentes de trabalho e desgastes físicos e psíquicos ${ }^{(4)}$.

Por definição, caracteriza-se como acidente de trabalho, os eventos ocorridos durante o desenvolvimento do trabalho que geram danos físicos ou mentais aos trabalhadores, de forma temporária, permanente ou fatal, ocasionando limitação funcional ou invalidez ${ }^{(5)}$. O trabalho no SAMU apresenta características que favorecem a ocorrência de acidentes de trabalho, devido as particularidades do trabalho dentro da ambulância, que apresenta espaço limitado, com pouca ventilação, dinâmica dos movimentos de tráfego, curvas acentuadas, assim como, o tipo de atendimento, que envolve situação de estresse pelas situações de emergência e necessidades de procedimentos para manutenção da vida dos pacientes ${ }^{(6)}$.

A identificação dos acidentes de trabalho é um fator que demanda atenção, tendo em vista que os trabalhadores pré-hospitalares estão expostos a diferentes tipos de riscos ocupacionais ${ }^{(6)}$. No atendimento pré-hospitalar, os trabalhadores do SAMU estão expostos a mais riscos ocupacionais do que os trabalhadores que desenvolvem atividades no ambiente hospitalar, uma vez que os atendimentos das vítimas ocorrem em diferentes locais e nas mais diferentes circunstâncias ${ }^{(7)}$.

Os riscos ocupacionais são decorrentes de fatores específicos do ambiente e condições de trabalho, assim como, características como o tempo de serviço, formação, categoria profissional. Entre os riscos ocupacionais cita-se o contato com fluidos como sangue e secreções, microrganismos como vírus e bactérias, contato com material perfurocortante, entre outros geradores de acidentes de trabalho durante o desenvolvimento das atividades laborais ${ }^{(8)}$.
Além disso, dada à complexidade do trabalho desenvolvido e a presença de riscos que podem ocasionar o adoecimento dos trabalhadores do ambiente pré-hospitalar, acidentes e até a morte, são necessárias medidas voltadas a saúde destes ${ }^{(2)}$. Entretanto, as ações de atenção à saúde dos trabalhadores ainda são pouco desenvolvidas pelos gestores dos serviços ou até mesmo inexistentes ${ }^{(9)}$. Entre os fatores que podem gerar melhores condições de trabalho está a redução de carga horária, dimensionamento de pessoal adequado e disponibilidade de insumos materiais suficientes para o desenvolvimento da assistência ${ }^{(2)}$.

Neste sentido, justifica-se a realização deste estudo frente à especificidade do serviço pré-hospitalar, no qual exige uma atenção constante, agilidade e conhecimento para realização do atendimento aos pacientes, vítimas e população nas distintas situações causais. Salienta-se ainda, que o trabalho desenvolvido pela equipe de pré-hospitalar possui alta relevância social, por ser o primeiro atendimento a ser realizado por uma equipe preparada, o que contribui para a sobrevivência e estabilidade do paciente ou vítima atendida.

Destacam-se além das especificidades do serviço, os distintos ambientes em que o trabalhador exerce o atendimento como, vias públicas, domicílios e locais de difícil acesso, que expõe o trabalhador a distintos riscos ocupacionais. Desta forma, este estudo pode colaborar com a reflexão acerca da implementação de estratégias e ações que minimizem a exposição aos riscos ocupacionais que possam causar a ocorrência de acidentes de trabalho, conjuntamente com as secretarias Municipais de Saúde e gestores do Serviço de Atendimento Móvel de Urgência dos municípios envolvidos no estudo.

Diante do exposto, este estudo teve como objetivo: analisar a ocorrência de acidentes de trabalho entre trabalhadores do Serviço de Atendimento Móvel de Urgência e a associação com os riscos ocupacionais identificados.

\section{MÉTODO}

\section{Desenho do estudo}

Trata-se de um estudo quantitativo, exploratório e descritivo, com delineamento transversal.

\section{PopulaÇão}

Estudo realizado com trabalhadores do SAMU 192, distribuídos em 57 municípios do estado do Rio Grande do Sul, com população de 1352 trabalhadores, compreendendo condutores de veículos de emergência, técnicos e auxiliares de enfermagem, enfermeiros e médicos. Os municípios participantes foram agrupados por mesorregiões, para melhor apresentação dos resultados, de acordo com o padrão adotado pelo Instituto Brasileiro de Geografia e Estatística (IBGE), conforme figura 1. 


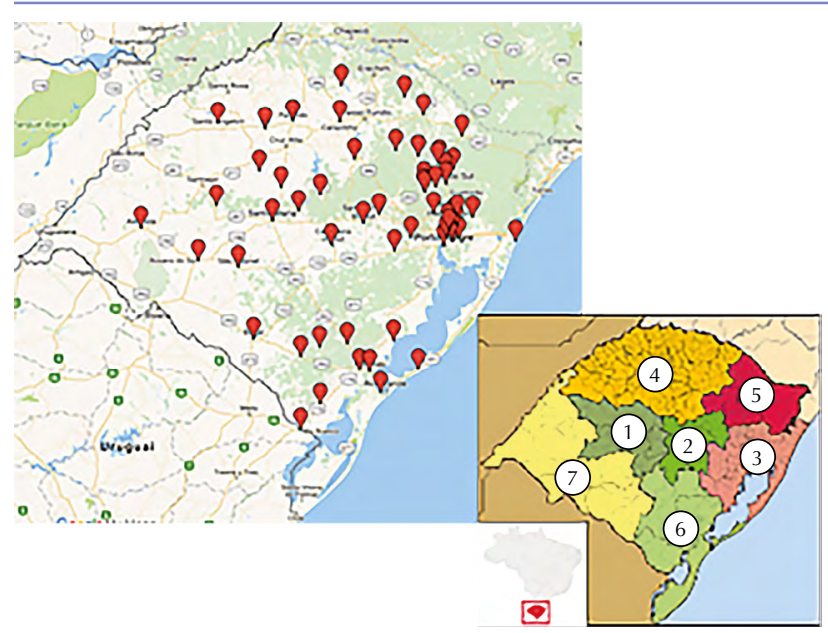

Legenda: 1 (Centro Ocidental Rio-Grandense); 2 (Centro Oriental Rio-Grandense); 3 (Metropolitana de Porto Alegre); 4 (Nordeste RioGrandense); 5 (Noroeste Rio-Grandense); 6 (Sudeste Rio-Grandense); 7 (Sudoeste Rio-Grandense).

Figura 1 - Distribuição geográfica dos municípios respondentes à pesquisa por local e por agrupamento em mesorregiões - Rio Grande, RS, Brasil, 2018.

Para o cálculo amostral utilizou-se o software EPI Info ${ }^{\mathrm{TM}}$ 7.2.2.6, a partir da população de 1352 trabalhadores, nível de confiabilidade de $90 \%$ e margem de erro amostral de $5 \%$, obteve-se a amostra mínima de 225 participantes. Participaram deste estudo, 265 trabalhadores, distribuídos de acordo com as categorias profissionais: 72 condutores de ambulância, quatro condutores de motolância, 83 enfermeiros, 87 técnicos ou auxiliares de enfermagem e 19 médicos.

\section{CRITÉRIOS DE SELEÇ̃̃o}

Foram convidados para participar do estudo, trabalhadores que atendessem os seguintes critérios de inclusão: possuir qualquer tipo vínculo de trabalho com Serviço de Atendimento Móvel de Urgência - SAMU 192, independente do tempo de trabalho e de desenvolver o processo de trabalho nas unidades móveis (ambulâncias e motolâncias). Excluíram-se do estudo, os pilotos de ambulanchas e de aeronaves, considerando que estes dispositivos não estão operantes no estado do Rio Grande do Sul e os profissionais que atuam exclusivamente em funções administrativas ou nas Centrais de Regulação Médica.

\section{Coleta de dados}

Os dados foram coletados no período entre janeiro de 2016 e novembro de 2017 por meio de um instrumento digital, através da plataforma gratuita do Google Forms e aplicado de forma online. $\mathrm{O}$ instrumento compreendeu questões sociodemográficas, ocorrência de acidentes de trabalho, frequência da presença de riscos ocupacionais e afastamentos do trabalho relacionados ao trabalho.
Realizou-se contato prévio com as secretarias municipais de saúde e gestores dos serviços de Serviço de Atendimento Móvel de Urgência para obtenção de autorização para o desenvolvimento do estudo e obtenção da relação de e-mails dos trabalhadores. Posteriormente, foi enviado o convite para a participação do estudo, juntamente com o link para acessar o instrumento individualmente e cópia do Termo de Consentimento Livre e Esclarecido a toda população do estudo.

\section{ANÁlISE E TRATAMENTO DOS DADOS}

Foram utilizados os softwares Microsoft Excel 2013 para tabulação dos dados, por meio da dupla digitação e o Statistical Package for the Social Sciences (SPSS) v.24 para a análise estatística dos dados. Realizou-se análise estatística descritiva a partir de frequência absoluta e relativa para caracterização dos participantes e ocorrência de acidentes. Para verificar as associações estatísticas entre a ocorrência de acidentes de trabalho e a categoria profissional, os acidentes de trabalho e a mesorregião de atuação dos trabalhadores foi utilizado o teste de Kruskal-Wallis e para associação entre a ocorrência de acidentes de trabalho e exposição de riscos ocupacionais utilizou-se o Teste $\chi 2 \mathrm{de}$ Pearson. O nível de significância estatística para todos os testes foi de $p>0,05$.

\section{Aspectos étICOS}

O estudo foi aprovado pelo Comitê de Ética em Pesquisa na Área da Saúde sob o Parecer n ${ }^{\circ}$. 118/2015 e atendeu aos aspectos éticos conforme Resolução 466/12, do Conselho Nacional de Saúde. Este estudo foi desenvolvido com a coparticipação da Secretaria Estadual de Saúde do Estado do Rio Grande do Sul através do Departamento de Regulação estadual do SAMU 192.

\section{RESULTADOS}

Entre os 265 trabalhadores participantes do estudo, 72 $(27,2 \%)$ eram condutores de ambulância, quatro $(1,5 \%)$ condutores de motolância, 83 (31,3\%) enfermeiros, 87 $(32,8 \%)$ técnicos ou auxiliares de enfermagem e $19(7,2 \%)$ médicos. Com relação ao sexo, verificou-se que $142(53,6 \%)$ eram do sexo masculino e $123(46,4 \%)$ do sexo feminino. A faixa etária predominante compreende de 30 a 45 anos, com $198(74,7 \%)$ trabalhadores, seguida de $31(11,7 \%)$ trabalhadores com idade inferior a 29 anos e $36(13,6 \%)$ acima de 45 anos.

Com relação à ocorrência de acidentes de trabalho, identificou-se que 139 (52,5\%) já sofreram algum tipo de acidente de trabalho durante suas atividades ocupacionais no ambiente pré-hospitalar. Na tabela 1, apresenta-se a associação significativa entre a ocorrência de acidente de trabalho e a categoria profissional $(\mathrm{p}=0,041)$, identificando a associação entre quedas no ambiente de trabalho com a categoria profissional $(\mathrm{p}=0,038)$. 
Tabela 1 - Relação entre a ocorrência de acidentes de trabalho e a categoria profissional - Rio Grande, RS, Brasil, 2018.

\begin{tabular}{|c|c|c|c|c|c|c|c|c|c|c|c|}
\hline \multirow[b]{3}{*}{ Variável } & \multicolumn{10}{|c|}{ Categoria profissional } & \multirow[b]{3}{*}{$\mathbf{p}$} \\
\hline & \multicolumn{2}{|c|}{$\begin{array}{l}\text { Enfermeiros } \\
\quad(n=83)\end{array}$} & \multicolumn{2}{|c|}{$\begin{array}{c}\text { Téc. / Aux. } \\
\text { Enfermagem }(n=87)\end{array}$} & \multicolumn{2}{|c|}{$\begin{array}{l}\text { Médicos } \\
(n=19)\end{array}$} & \multicolumn{2}{|c|}{$\begin{array}{c}\text { Condutores } \\
\text { ambulância }(n=72)\end{array}$} & \multicolumn{2}{|c|}{$\begin{array}{c}\text { Condutores } \\
\text { motolância }(n=4)\end{array}$} & \\
\hline & $\mathbf{n}$ & $\%$ & $\mathbf{n}$ & $\%$ & n & $\%$ & $\mathbf{n}$ & $\%$ & $\mathbf{n}$ & $\%$ & \\
\hline Acidente de trabalho & 38 & 45,8 & 56 & 64,4 & 11 & 57,9 & 31 & 43,1 & 3 & 75 & 0,041 \\
\hline \multicolumn{12}{|l|}{ Tipos de acidentes } \\
\hline Perfurocortantes & 17 & 20,5 & 23 & 26,4 & 2 & 10,5 & 11 & 15,3 & 2 & 50 & 0,179 \\
\hline Agressão física & 9 & 10,8 & 20 & 23 & 4 & 21,1 & 8 & 11,1 & 1 & 25 & 0,152 \\
\hline Mordida de animais & 7 & 8,4 & 9 & 10,3 & 2 & 10,5 & 4 & 5,6 & 1 & 25 & 0,624 \\
\hline Agressão verbal & 30 & 36,1 & 43 & 48,4 & 11 & 57,9 & 23 & 31,9 & 2 & 50 & 0,087 \\
\hline Acidentes de trânsito no deslocamento & 7 & 8,4 & 17 & 19,5 & 1 & 5,3 & 9 & 12,5 & 1 & 25 & 0,182 \\
\hline Atropelamento na cena de atendimento & 1 & 1,2 & 1 & 1,1 & 0 & 0 & 0 & 0 & 0 & 0 & 0,891 \\
\hline Quedas & 15 & 18,1 & 21 & 24,1 & 5 & 26,3 & 6 & 8,3 & 2 & 50 & 0,038 \\
\hline Queimadura por produtos químicos & 0 & 0 & 1 & 1,1 & 1 & 5,3 & 0 & 0 & 0 & 0 & 0,163 \\
\hline
\end{tabular}

Teste Kruskal Wallis; $\mathrm{p}>0,05$.

Também se identificou associação significativa entre a ocorrência de acidente de trabalho e a mesorregião $(p=0,015)$ e associação estatisticamente significativa entre a mesorregião de atuação do trabalhador com acidente com perfurocortante $(p=0,016)$, mordida de animal $(p=0,016)$, quedas $(p=0,002)$ e queimadura com fogo $(\mathrm{p}=0,024)$, conforme a Tabela 2 .

Tabela 2 - Relação entre a ocorrência de acidentes de trabalho de acordo a mesorregião de atuação dos trabalhadores - Rio Grande, RS, Brasil, 2018.

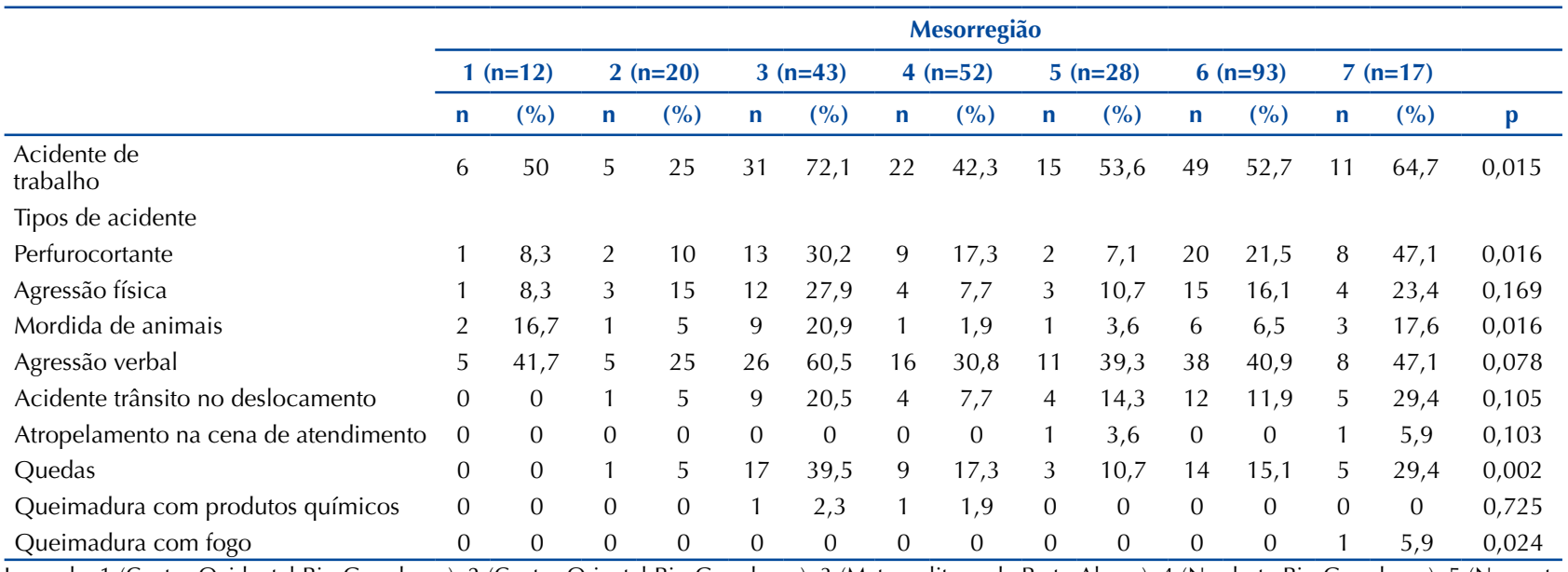

Legenda: 1 (Centro Ocidental Rio-Grandense); 2 (Centro Oriental Rio-Grandense); 3 (Metropolitana de Porto Alegre); 4 (Nordeste Rio-Grandense); 5 (Noroeste Rio-Grandense); 6 (Sudeste Rio-Grandense); 7 (Sudoeste Rio-Grandense). Teste Kruskal Wallis; $p>0,05$.

$\mathrm{Na}$ Tabela 3 identificam-se as associações entre a ocorrência de acidentes de trabalho e os riscos ocupacionais identificados pelos trabalhadores. A ocorrência de acidente de trabalho apresentou associação significativa com os seguintes riscos ocupacionais: postura inadequada $(\mathrm{p}=0,012)$; jornada de trabalho prolongada $(\mathrm{p}=0,001)$; estresse $(\mathrm{p}=0,000)$; sobrecarga de trabalho $(\mathrm{p}=0,002)$; iluminação inadequada $(\mathrm{p}=0,000)$; ansiedade $(\mathrm{p}=0,000)$; esgotamento físico $(\mathrm{p}=0,000)$; esgotamento psíquico $(\mathrm{p}=0,000)$; arranjo físico inadequado ( $\mathrm{p}=0,003)$; máquinas e equipamentos sem proteção $(\mathrm{p}=0,006)$; equipamentos defeituosos $(\mathrm{p}=0,013)$.

Os acidentes com perfurocortantes apresentaram associação estatisticamente significativa com os riscos jornada de trabalho prolongada $(\mathrm{p}=0,038)$; estresse $(\mathrm{p}=0,032)$; sobrecarga de trabalho ( $\mathrm{p}=0,028)$; iluminação inadequada $(\mathrm{p}=0,001)$; ansiedade ( $\mathrm{p}=0,005)$; esgotamento físico $(\mathrm{p}=0,013)$; esgotamento psíquico $(\mathrm{p}=0,006)$; arranjo físico inadequado $(\mathrm{p}=0,001)$ e máquinas e equipamentos sem proteção $(\mathrm{p}=0,001)$.

Com relação à agressão física no ambiente ocupacional verificou-se associação estatisticamente significativa com jornada de trabalho prolongada $(\mathrm{p}=0,007)$; estresse $(\mathrm{p}=0,000)$; sobrecarga de trabalho $(\mathrm{p}=0,000)$; iluminação inadequada $(\mathrm{p}=0,010)$; ansiedade $(\mathrm{p}=0,003)$; esgotamento físico $(\mathrm{p}=0,000)$; esgotamento psíquico $(\mathrm{p}=0,002)$; arranjo físico inadequado ( $\mathrm{p}=0,007)$; máquinas e equipamentos sem proteção ( $p=0,008)$; equipamentos defeituosos $(\mathrm{p}=0,029)$.

A ocorrência de mordidas de animais também apresentou associação estatisticamente significativa com ansiedade $(\mathrm{p}=0,008)$; esgotamento físico $(\mathrm{p}=0,028)$; esgotamento $\mathrm{psí}-$ quico $(\mathrm{p}=0,039)$. 
Considerando a ocorrência de agressão verbal identificou-se a associação estatisticamente significativa com postura inadequada $(\mathrm{p}=0,003)$; jornada de trabalho prolongada $(\mathrm{p}=0,011)$; estresse $(\mathrm{p}=0,000)$; sobrecarga de trabalho $(\mathrm{p}=0,003)$; iluminação inadequada $(\mathrm{p}=0,000)$; ansiedade $(\mathrm{p}=0,000)$; esgotamento físico $(\mathrm{p}=0,000)$; esgotamento psíquico $(\mathrm{p}=0,000)$; arranjo físico inadequado $(\mathrm{p}=0,001)$; máquinas e equipamentos sem proteção $(\mathrm{p}=0,001)$; equipamentos defeituosos $(\mathrm{p}=0,001)$.
Já o acidente de trânsito no deslocamento mostrou associação significativa com jornada de trabalho prolongada $(\mathrm{p}=0,022)$; iluminação inadequada $(\mathrm{p}=0,000)$; ansiedade $(\mathrm{p}=0,041)$; esgotamento físico $(\mathrm{p}=0,023)$; e esgotamento psíquico $(\mathrm{p}=0,013)$. Enquanto as quedas apresentam associação estatisticamente significativa com postura inadequada $(\mathrm{p}=0,022)$; jornada de trabalho prolongada ( $\mathrm{p}=0,027)$; estresse $(\mathrm{p}=0,016)$; iluminação inadequada $(0,029)$; ansiedade $(\mathrm{p}=0,007)$; esgotamento físico $(0,037)$; esgotamento psíquico ( $\mathrm{p}=0,002)$; e, equipamentos defeituosos $(\mathrm{p}=0,018)$.

Tabela 3 - Associação entre a ocorrência de acidentes ocupacionais e o risco ocupacional observado pelo trabalhador - Rio Grande, RS, Brasil, 2018.

\begin{tabular}{|c|c|c|c|c|c|c|c|}
\hline \multirow{3}{*}{ Riscos ocupacionais } & \multirow[b]{2}{*}{$\begin{array}{l}\text { Ocorrência de } \\
\text { acidente de } \\
\text { trabalho }\end{array}$} & \multicolumn{6}{|c|}{ Tipos de acidentes de trabalho } \\
\hline & & $\begin{array}{l}\text { Perfuro } \\
\text { cortante }\end{array}$ & $\begin{array}{l}\text { Agressão } \\
\text { física }\end{array}$ & $\begin{array}{l}\text { Mordida de } \\
\text { animais }\end{array}$ & $\begin{array}{l}\text { Agressão } \\
\text { verbal }\end{array}$ & $\begin{array}{c}\text { Acidente } \\
\text { trânsito no } \\
\text { deslocamento }\end{array}$ & Quedas \\
\hline & p-valor & p-valor & p-valor & p-valor & p-valor & p-valor & p-valor \\
\hline Postura inadequada & 0,012 & 0,452 & 0,125 & 0,364 & 0,003 & 0,123 & 0,022 \\
\hline Trabalho em turno e noturno & 0,053 & 0,786 & 0,074 & 0,721 & 0,300 & 0,182 & 0,197 \\
\hline Jornada de trabalho prolongada & 0,001 & 0,038 & 0,007 & 0,682 & 0,011 & 0,022 & 0,027 \\
\hline Estresse & 0,000 & 0,032 & 0,000 & 0,188 & 0,000 & 0,062 & 0,016 \\
\hline Sobrecarga de trabalho & 0,002 & 0,028 & 0,000 & 0,671 & 0,003 & 0,101 & 0,152 \\
\hline Iluminação inadequada & 0,000 & 0,001 & 0,010 & 0,085 & 0,000 & 0,000 & 0,029 \\
\hline Ansiedade & 0,000 & 0,005 & 0,003 & 0,008 & 0,000 & 0,041 & 0,007 \\
\hline Esgotamento físico & 0,000 & 0,013 & 0,000 & 0,028 & 0,000 & 0,023 & 0,037 \\
\hline Esgotamento psíquico & 0,000 & 0,006 & 0,002 & 0,039 & 0,000 & 0,013 & 0,002 \\
\hline Arranjo físico inadequado & 0,003 & 0,001 & 0,007 & 0,321 & 0,001 & 0,154 & 0,347 \\
\hline Máquinas e equipamentos sem proteção & 0,006 & 0,001 & 0,008 & 0,434 & 0,001 & 0,256 & 0,409 \\
\hline Equipamentos defeituosos & 0,013 & 0,004 & 0,029 & 0,569 & 0,001 & 0,177 & 0,018 \\
\hline
\end{tabular}

Teste $\chi 2$ de Pearson; $\mathrm{p}>0,05$

Com relação aos afastamentos de trabalho, $39(14,7 \%)$ trabalhadores já precisaram ficar afastados por algum acidente de trabalho, destes, 20 (7,5\%) ficaram afastados até sete dias, $14(5,3 \%)$ afastados de sete a 14 dias, dois $(0,8 \%)$ de 15 a 30 dias, três $(1,1 \%)$ de 30 a 60 dias, dois $(0,8 \%)$ trabalhadores de 60 a 180 dias e três $(1,1 \%)$ mais de 180 dias. Além disso, foi verificada a associação significativa entre a ocorrência de acidente de trabalho e os afastamentos do trabalho $\left(\mathrm{X}^{2}=41,453 ; \mathrm{gl}=1 ; \mathrm{p}=0,000\right)$.

\section{DISCUSSÃO}

A ocorrência de acidentes de trabalho durante o desenvolvimento das atividades no ambiente pré-hospitalar foi identificada por 139 (52,5\%) trabalhadores, tal dado vai ao encontro a um estudo realizado em uma Central de SAMU em uma cidade de Paraíba, com 37 trabalhadores, dos quais, $30 \%$ referiram já ter sofrido acidente de trabalho anteriormente ${ }^{(6)}$.

Os acidentes de trabalho apresentaram associação estatisticamente significativa com a categoria profissional dos trabalhadores do SAMU. Entre os acidentes de trabalho, destaca-se que o acidente com perfurocortante foi o identificado como o de maior frequência entre os trabalhadores, este tipo de acidente de trabalho expõe o trabalhador a fluídos biológicos. No serviço pré-hospitalar do município de Goiânia, verificou-se que a categoria profissional que mais sofreu acidentes de trabalho com exposição a material biológico foi os enfermeiros, seguidos pelos médicos, técnicos em enfermagem e condutores de ambulâncias ${ }^{(10)}$. Essa característica pode estar relacionada à frequente presença do enfermeiro na realização dos atendimentos. Em estudo ${ }^{(11)}$ em uma Central do SAMU em Cuiabá, verificou-se que de 1007 atendimentos realizados pela unidade, em 926 (91,9\%) o enfermeiro estava presente durante a assistência.

Também se verificou associação estatisticamente significativa entre acidentes de trabalho e a mesorregião em que os trabalhadores desenvolviam suas atividades, entre os acidentes, destacam-se os acidentes com perfurocortantes, mordidas de animal, quedas e queimaduras com fogo. $\mathrm{Na}$ análise de atendimentos por queimaduras em um pronto socorro na cidade de Pelotas, Rio Grande do Sul, verificou-se que dos 529 atendimentos queimaduras realizados pelo serviço, o local predominante de ocorrência do acidente foi no trabalho e no ambiente domiciliar, sendo o SAMU, o serviço que conduziu $5 \%$ destes pacientes ao Pronto Socorro ${ }^{(12)}$, o atendimento realizado pelo SAMU a estas vítimas pode expor os trabalhadores aos acidentes de trabalho.

Verificou-se associação estatisticamente significativa entre a ocorrência de acidentes de trabalho e os riscos ocupacionais identificados por esses trabalhadores nos seus ambientes de trabalho. Entre os riscos ocupacionais citados, destacaram-se a postura inadequada, jornada de trabalho 
prolongada, estresse, sobrecarga de trabalho, iluminação inadequada, ansiedade, esgotamento físico, esgotamento psíquico, arranjo físico inadequado, máquinas e equipamentos sem proteção e equipamentos defeituosos. Estudo com 572 trabalhadores do Emergency Medical Services (EMS) identificou entre as lesões sofridas pelos trabalhadores, as entorses e distensões, lesões corporais decorrentes do levantamento, transporte e transferência de pacientes e equipamentos e as exposições a substâncias nocivas a saúde. Os trabalhadores ainda relacionaram estes acidentes de trabalho ao esforço físico, postura corporal inadequada, movimentos repetitivos, exposição a produtos químicos, assim como, escorregões, quedas, acidentes de trânsito e agressões ou violência ${ }^{(13)}$.

De acordo com a ocorrência de acidentes com material perfurocortantes sofridos pelos trabalhadores, verificou-se associação significativa com diversos riscos ocupacionais como a ansiedade, estresse, esgotamento físico, sobrecarga de trabalho e jornadas de trabalho prolongada. Os acidentes envolvendo perfurocortantes ocorrem principalmente durante a realização de punção venosa, glicemia capilar, medicação intradérmica e reencape da agulha, sutura, mediação intramuscular e retirada de cateter intravenoso. Além disso, os acidentes de trabalho estão associados à prática da assistência ao paciente no interior da ambulância em movimento, ou ainda, na cena onde o atendimento é realizado ${ }^{(14)}$. Mordidas de animais apresentou associação significativa com os riscos ocupacionais ansiedade e esgotamento físico e psíquico. Além das lesões decorrentes das mordidas, este acidente também é potencialmente capaz de transmitir material biológico contaminado aos trabalhadores, pois causam a transferência de micro-organismos patogênicos ${ }^{(6)}$.

As agressões físicas e verbais são caracterizadas como violência no ambiente de trabalho, a agressão verbal apresenta crescimento e é a que mais atinge os trabalhadores, ocorrendo especialmente pela insatisfação dos pacientes devida a dificuldade de acesso ao atendimento de saúde e déficits no acolhimento ${ }^{(15)}$. Quanto as agressões físicas sofridas durante o exercício laboral, identificou-se associação significativa com os riscos ocupacionais: sobrecarga de trabalho, estresse, esgotamento físico, esgotamento psíquico e arranjo físico inadequado. A tensão e estresse durante a assistência pré-hospitalar ocorrem normalmente em pacientes graves, psiquiátricos e agressivos $^{(16)}$. A agressão física entre os trabalhadores do ambiente pré-hospitalar é uma característica de frequência crescente, como aponta um estudo ${ }^{(7)}$ com 300 registros de acidentes graves que teve como principal mecanismo de lesão a agressão interpessoal, representando 62\% dos casos analisados.

Já no acidente agressão verbal, destacam-se as associações significativas com os seguintes riscos ocupacionais: postura inadequada, jornada de trabalho prolongada, estresse, sobrecarga de trabalho, iluminação inadequada, ansiedade, esgotamento físico, esgotamento psíquico, arranjo físico inadequado, máquinas e equipamentos sem proteção, equipamentos defeituosos. As agressões verbais são identificadas como insultos e xingamentos por parte da população ou do paciente, os quais também ocorrem com frequência durante a realização da assistência ${ }^{(17)}$, assim como o assédio relacionado ao trabalho ou o assédio moral no próprio ambiente de traba1 ho ${ }^{(7)}$. O abuso verbal foi investigado em estudo com 72 profissionais (enfermeiros, parteiras, cinesiologistas) e 148 técnicos paramédicos (auxiliares de enfermagem) de atendimento pré-hospitalar em três regiões no sul do Chile, verificando que entre os principais abusadores verbais, destacam-se os pacientes, seus familiares e o público em geral (80\%), e os colegas externos ao SAMU $(34,6 \%)^{(18)}$.

Com relação aos acidentes de trânsito durante o deslocamento dos veículos verificou-se associação significativa com a jornada de trabalho prolongada, iluminação inadequada, ansiedade e esgotamento físico e psíquico. Tal fato está relacionado às dificuldades enfrentadas no trânsito, sobretudo, o desrespeito às leis de trânsito e os buracos nas vias, fatores que desencadeiam estresse e aborrecimento entre os trabalhadores durante a assistência ${ }^{(19)}$. Além disso, o trabalho no SAMU expõem os trabalhadores a riscos ocupacionais, como tensão e estresse, desencadeados principalmente pelo trânsito e pelo ruído da sirene ${ }^{(16)}$, assim como, a incompreensão da população acerca da necessidade de deslocamento rápido das ambulâncias, gerando acidentes ${ }^{(7)}$. Destaca-se que os acidentes de trânsito envolvendo a ambulância e outros veículos são considerados frequentes na prática laboral, estudo ${ }^{(14)}$ mostra o envolvimento de $81,4 \%$ de 901 registros de acidentes laborais em pelo menos um acidente desta natureza.

As quedas apresentaram associação significativa com os riscos ocupacionais: postura inadequada, jornada de trabalho prolongada, estresse, iluminação inadequada, ansiedade, esgotamento físico, esgotamento psíquico e equipamentos defeituosos. Outro estudo ${ }^{(20)}$ identificou maior incidência de quedas decorrentes da exposição aos riscos ocupacionais, devido as características da realização de cuidados ininterruptos aos pacientes, fatores organizacionais e comportamentais dos trabalhadores.

A ocorrência de acidentes de trabalho também apresentou associação estatisticamente significativa com os afastamentos do trabalho, além disso, $14,7 \%$ dos trabalhadores referiram que já precisaram ficar afastados devido a algum acidente de trabalho. Em estudo com trabalhadores do EMS nos Estados Unidos analisou 950 registros de eventos envolvendo a saúde dos trabalhadores, 705 deles estavam relacionados a algum tipo de acidente de trabalho. Além disso, o estudo destaca que os impactos dos agravos na saúde destes trabalhadores refletem na necessidade de afastamento temporário e perdas de dias de trabalho, o que pode comprometer o desenvolvimento das atividades prestadas ${ }^{(21)}$.

Os resultados obtidos podem contribuir para o conhecimento científico e para a área da Enfermagem no que tange a discussão da prática assistencial no serviço pré-hospitalar, vislumbrando a segurança do trabalhador no decorrer do desenvolvimento das suas atividades e a exposição aos riscos ocupacionais geradores de acidentes de trabalho. Evidencia-se que embora o uso da tecnologia e redes sociais seja um avanço na área da pesquisa, neste estudo, o acesso aos participantes por meio digital foi uma limitação, pois foram verificadas dificuldades relacionadas a participação de trabalhadores de diferentes municípios do interior e da região metropolitana do Rio Grande do Sul. 


\section{CONCLUSÃO}

O estudo permitiu analisar a ocorrência de acidentes de trabalho entre trabalhadores da saúde do Serviço de Atendimento Móvel de Urgência e identificar associação estatisticamente significativa entre a ocorrência de acidentes de trabalho e a categoria profissional, tendo em vista que diferentes categorias profissionais desempenham atividades no ambiente pré-hospitalar e cada uma delas possui atribuições que as expõe a riscos e acidentes. Assim como, a associação entre a ocorrência de acidentes de trabalho com a mesorregião de atuação do trabalhador, indicando que cada região apresenta riscos de acordo com as características do local.

A identificação da associação significativa entre acidentes de trabalho com a presença de riscos ocupacionais e com os afastamentos do trabalho sugere a necessidade do planejamento de ações de prevenção para estes riscos e consequentemente para evitar os acidentes de trabalho. Neste sentido, espera-se promover reflexão sobre as práticas profissionais entre estes trabalhadores e gestores dos serviços, com o intuito de promover melhorias nas condições de trabalho e na segurança do trabalhador no ambiente de trabalho, com a finalidade de reduzir a ocorrência de acidentes de trabalho.

\section{RESUMO}

Objetivo: Analisar a ocorrência de acidentes de trabalho entre trabalhadores do Serviço de Atendimento Móvel de Urgência e a associação com os riscos ocupacionais identificados. Método: Estudo quantitativo, exploratório, descritivo e transversal, realizado com trabalhadores, entre janeiro de 2016 e novembro de 2017, por meio de um instrumento online, no Rio Grande do Sul. Resultados: Participaram 265 trabalhadores. Verificou-se associação significativa entre ocorrência de acidente de trabalho e categoria profissional $(p=0,041)$; ocorrência de acidente de trabalho e a mesorregião $(p=0,015)$. Nas associações significativas entre a ocorrência de acidentes de trabalho e os riscos ocupacionais, destacaram-se os acidentes com perfurocortantes, agressão física, mordida de animal, agressão verbal, acidente de trânsito no deslocamento e quedas. Assim como, a associação significativa entre a ocorrência de acidente de trabalho e os afastamentos do trabalho $(\mathrm{p}=0,000)$. Conclusão: As ocorrências de acidentes de trabalho durante as atividades no serviço préhospitalar estão relacionadas à categoria profissional dos trabalhadores, mesorregião de atuação, afastamentos do trabalho e a exposição dos trabalhadores a distintos riscos ocupacionais.

\section{DESCRITORES}

Acidentes de Trabalho; Serviços Médicos de Emergência; Ambulância; Riscos Ocupacionais; Saúde do Trabalhador.

\section{RESUMEN}

Objetivo: Analizar la ocurrencia de accidentes de trabajo entre trabajadores del Servicio de Atendimiento Móvil de Urgencia y la asociación con los riscos ocupacionales identificados. Método: Estudio cuantitativo, exploratorio, descriptivo y transversal, realizado con trabajadores, entre enero de 2016 y noviembre de 2017, por medio de un instrumento online, no Rio Grande do Sul. Resultados: Participaran 265 trabajadores. Verifico-se asociación significativa entre ocurrencia de accidente de trabajo y categoría profesional ( $p=0,041)$; ocurrencia de accidente de trabajo y la mesorregión $(\mathrm{p}=0,015)$. En las asociaciones significativas entre la ocurrencia de accidentes de trabajo y los riscos ocupacionales, destacaran-se los accidentes con objetos punzantes, agresión física, mordida de animal, agresión verbal, accidente de tránsito en el dislocamiento y quedas. Así como, la asociación significativa entre la ocurrencia de accidente de trabajo y las retiradas del trabajo $(\mathrm{p}=0,000)$. Conclusión: Las ocurrencias de accidentes de trabajo durante las actividades en el servicio pre-hospitalario están relacionadas a la categoría profesional de los trabajadores, mesorregión de actuación, retirada del trabajo y la exposición de los trabajadores a distintos riscos ocupacionales.

\section{DESCRIPTORES}

Accidentes de Trabajo; Servicios Médicos de Urgencia; Ambulancias; Riesgos Laborales; Salud Laboral.

\section{REFERÊNCIAS}

1. Gomes BB, dos Santos WL. Acidentes laborais entre equipe de atendimento pré-hospitalar móvel (Bombeiros/SAMU) com destaque ao risco biológico. Revisa Rev Divulg Cien Sena Aires [Internet]. 2012 [citado 2019 maio 07];1(1):40-9. Disponível em: http://revistafacesa. senaaires.com.br/index.php/revisa/article/view/11

2. Silva OM, Ascari RA, Schiavinato D, Ribeiro MC. Riscos de adoecimento enfrentados pela equipe de enfermagem do SAMU: uma revisão integrativa. Rev Saúde Pública Santa Catarina [Internet]. 2014 [citado 2019 maio 07];1(1):107-21. Disponível em: http://revista.saude. sc.gov.br/index.php/inicio/article/view/172/249

3. Brasil. Ministério da Saúde. Portaria n. 2048, de 5 de novembro de 2002. Aprova o regulamento técnico dos sistemas estaduais de urgência e emergência [Internet]. Brasília, 2002 [citado 2019 maio 07]. Disponível em: http://bvsms.saude.gov.br/bvs/saudelegis/gm/2002/ prt2048_05_11_2002.html

4. Nascimento MO, Araújo GF. Riscos ocupacionais dos profissionais de enfermagem atuantes no SAMU 192. Rev Multidisc Psicol [Internet] 2017 [citado 2019 maio 07];10(33). Disponível em: https://idonline.emnuvens.com.br/id/article/view/614/864

5. Brasil. Lei n. 8.213, de 24 de julho de 1991. Dispõe sobre os planos de benefícios da Previdência Social e dá outras providências [Internet]. Brasília; 1991 [citado 2019 maio 07]. Disponível em: http://www.planalto.gov.br/ccivil_03/LEIS/L8213cons.htm

6. Souza ND, Pinheiro MBGN, Moésia RV. Acidentes ocupacionais em profissionais de saúde no atendimento pré-hospitalar. J Med Health Prom [Internet]. 2016 [citado 2019 maio 07];1(1):1-10. Disponível em: http://jmhp.fiponline.edu.br/pdf/cliente=13-df68e972bf501ee04 f23fb8c9ae36cd9.pdf

7. Leite HDCS, Carvalho MTR, Cariman SLS, Araújo ERM, Silva NC, Carvalho AO. Risco ocupacional entre profissionais de saúde do Serviço de Atendimento Móvel de Urgência - SAMU. Enferm Foco [Internet]. 2016 [citado 2019 maio 07];7(3/4):31-5. Disponível em: http:// revista.cofen.gov.br/index.php/enfermagem/article/view/912/342

8. Couto PLS, Gomes AC, Alves FF, Castelan E, Dib RV, Mercês MC, et al. Representações sociais acerca dos riscos de acidentes de trabalho. Rev Bras Promoç Saúde [Internet]. 2018 [citado 2019 jul. 15];31(2):1-10. Disponível em: https://periodicos.unifor.br/RBPS/article/view/7074 
9. Mesquita KL, Gomes GPLA, Silva MJBF, Santos LF. A visão do enfermeiro/gestor sobre a necessidade de implementar apoio psicológico aos profissionais do serviço de atendimento móvel de urgência. Rev Enferm Cent Oeste Min [Internet]. 2014 [citado 2018 dez. 17];4(1):101928. Disponível em:

10. Tipple AFV, Silva EAC, Teles SA, Mendonça KM, Souza ACS, Melo DS. Acidente com material biológico no atendimento pré-hospitalar móvel: realidade para trabalhadores da saúde e não saúde. Rev Bras Enferm [Internet]. 2013 [citado 2019 maio 09];66(3):378-84. Disponível em: http://www.scielo.br/pdf/reben/v66n3/a12v66n3.pdf

11. Ribeiro AC, Silva YB. Enfermagem pré-hospitalar no suporte básico de vida: postulados ético-legais da profissão. Cogitare Enferm. [Internet]. 2016 [citado 2019 maio 09];21(1):1-8. Disponível em: http://docs.bvsalud.org/biblioref/2016/07/704/42118-171302-1-pb.pdf

12. Bartel TE, Sabia-Sturbelle C, Bazzan JS, Echavarria-Guanilo ME, Ceolin T. Análises dos registros dos atendimentos por queimaduras em uma unidade de urgência e emergência. Rev Enferm UFPE [Internet]. 2016 [citado 2019 maio 09];10(7):2345-53. Disponível em: https:// periodicos.ufpe.br/revistas/revistaenfermagem/article/viewFile/11289/12944

13. Reichard AA, Marsh SM, Tonozzi TR, Konda S, Gormley MA. Occupational injuries and exposures among Emergency Medical Services workers. Prehosp Emerg Care. 2017;21(4):420-31. DOI: http://dx.doi.org/10.1080/10903127.2016.1274350

14. Gülen B, Serinken M, Hatipoğlu C, Özaşır D, Sönmez E, Kaya G, et al. Work-related injuries sustained by emergency medical technicians and paramedics in Turkey. Ulus Travma Acil Cerrahi Derg. 2016;22(2):145-9.

15. Almeida NR, Bezerra Filho JG, Marques LA. Análise da produção científica sobre a violência no trabalho em serviços hospitalares. Rev Bras Med Trab. 2017;15(1):101-12. DOI: http://dx.doi.org/10.5327/Z1679443520177029

16. Costa IKF, Liberato SMD, Costa IKF, Melo MDM, Simpson CA, Farias GM. Ocupational hazards in a mobile emergency care. Rev Online Pesq Cuid Fundam [Internet]. 2013 [cited 2018 Dec 17];6(3):938-47. Available from: http://www.seer.unirio.br/index.php/cuidadofundamental/ article/view/3250/pdf_1336

17. Oliveira AC, Paiva MHRS. Analysis of occupational accidents with biological material among professionals in pre-hospital services. Rev Latino Am Enfermagem [Internet]. 2013 [cited 2018 Dec 17];21(1):309-15. Available from: http://www.scielo.br/pdf/rlae/v21n1/v21n1 a04.pdf

18. Rodríguez-Campo VA, Paravic-Klijn TM. Verbal abuse and mobbing in pre-hospital care services, Chile. Rev Latino Am Enfermagem. 2017;25:e2956. DOI: http://dx.doi.org/10.1590/1518-8345.2073.2956

19. Maia EC, Miranda MDC, Caetano JA, Carvalho MF, Santos MCL, Caldini LN. Evaluation of the level of stress of the nursing of mobile emergence care service. Rev Online Pesq Cuid Fundam [Internet]. 2012 [cited 2019 May 07];4(4):3060-8. Available from: http://www. seer.unirio.br/index.php/cuidadofundamental/article/view/1885/pdf_664

20. Rezende LCM, Leite KNS, Santos SR, Monteiro LC, Costa MBS, Santos FX. Acidentes de trabalho e suas repercussões na saúde dos profissionais de enfermagem. Rev Baiana Enferm. 2015;29(4):307-17. DOI: http://dx.doi.org/10.18471/rbe.v29i4.13559

21. Weaver MD, Patterson PD, Fabio A, Moore CG, Freiberg MS, Songer TJ. An observational study of shift length, crew familiarity, and occupational injury and illness in emergency medical services workers. Occup Environ Med [Internet]. 2015 [cited 2019 May 07];72:798804. Available from: https://www.ncbi.nlm.nih.gov/pmc/articles/PMC4686303/ 